Ananya Dwivedi and Ram Keval*

\title{
Analysis for transmission of dengue disease with different class of human population
}

https://doi.org/10.1515/em-2020-0046

Received October 28, 2020; accepted April 5, 2021; published online June 1, 2021

\begin{abstract}
Objectives: Vector-borne diseases speedily infest the human population. The control techniques must be applied to such ailment and work swiftly. We proposed a compartmental model of dengue disease by incorporating the standard incidence relation between susceptible vectors and infected humans to see the effect of manageable parameters of the model on the basic reproduction number.

Methods: We compute the basic reproduction number by using the next -generation matrix method to study the local and global stability of disease free and endemic equilibrium points along with sensitivity analysis of the model.

Results: Numerical results are explored the global behaviourism of disease-free/endemic state for a choice of arbitrary initial conditions. Also, the biting rate of vector population has more influence on the basic reproduction number as compared the other parameters.

Conclusion: In this paper, shows that controlling the route of transmission of this disease is very important if we plan to restrict the transmission potential.
\end{abstract}

Keywords: basic reproduction number $\left(R_{0}\right)$; dengue fever; numerical simulation; sensitivity; stability.

\section{Introduction}

One of the major concerns of the present era is the birth of a new class of diseases, especially vectorborne diseases like Japanese encephalitis, dengue, malaria, yellow fever etc. Earlier, the sources of these diseases were limited. But, new sources have evolved to a similar kind of ailment, which critically hits the human population. Therefore, one of the significant ways to understand the dynamic phenomenon of disease transmission is mathematical modelling. It helps in determining the intervention techniques for disease control and reduce the mortality rate of humans. Nowadays, a common viral disease in the tropical region of the world is dengue. Previously, it is known as dengue hemorrhagic fever. In the 1950s, the Philippines and Thailand countries were plagued, dengue epidemics and recognized for the first time. Currently, Latin, American and Asian countries are the most affected ones causing hospitalization and death of people from almost all age groups. Here, the mosquitoes act as transmitters, and humans act as receiver, describing the life cycle of the dengue virus (DENV). Aedes mosquito is the transmitter of dengue after an infectious bite by anyone of the given dengue virus (DENV-1 to 4). Symptoms of dengue appear in 3-14 days after the infectious bite of mosquitoes. Mostly, adult and young children are affected by viral dengue. Dengue causes grievous fever-like illness due to mosquito-borne viral infection. Sometimes, dengue becomes a potentially lethal

*Corresponding author: Ram Keval, Department of Mathematics and Scientific Computing, Madan Mohan Malaviya University of Technology, Gorakhpur, 273010, Uttar Pradesh, India, E-mail: ramkeval@gmail.com

Ananya Dwivedi, Department of Mathematics and Scientific Computing, Madan Mohan Malaviya University of Technology, Gorakhpur, Uttar Pradesh, India 
convolution. The incidence of dengue has increased 30-fold over the last 50 years. Distinct serotypes (DENV-1 to 4) are the disease origin, belonging to the genus Flavivirus family. If the human recovered from dengue virus, they are never affected again by the virus and receive immunity for a lifetime against that specific virus.

Only partial and momentary protection produced such immunity against the infection due to distinct serotypes of the virus. In comparison to transmitter, mosquitoes have the only selective method to eradicate the dengue virus. According to WHO (World Health Organisation), the estimation of dengue infections worldwide touches the range of 50-100 million, subjecting nearly half of the population all over the world at grave risk. There is no specific vaccine available for dengue. One recent observation in 2012 predicted that 3.9 billion people of around 128 countries are at potential risk of infection with DENV. Another contemplation in 2013 indicates that out of 390 million, 96 million (67-136 million) people infected through dengue per year and manifest clinically (with any severity of disease) (WHO 2020).

To research the mechanisms of dengue transmission, several mathematical models have been proposed and studied from Ghosh, Tiwari, and Chttopadhyay (2019) to Agusto and Khan (2018). In Ghosh, Tiwari, and Chttopadhyay (2019), the authors analyzed a compartmental model and studied dynamic behaviour of the model. Also, discuss ACF (active case finding) for asymptomatic and symptomatic individuals plays a crucial role in reducing disease prevalence. In Zhu and $\mathrm{Xu}$ (2019) studied an SIS-SI dengue model associated with a periodic environment to discuss temporal periodicity and spatial heterogeneity. Driessch and Watmough (2002) utilized a system of ODE to formulate a compartmental model for disease transmission and analyzed the stability and instability at $R_{0}<1$ and $R_{0}>1$ respectively. In Busenberg and Driessch (1990) and Esteva and Vargas (1999), the authors analyzed a dengue transmission model with varying size and applied competitive system theory to discuss the equilibrium's global asymptotic stability. Esteva and Vargas $(1998,2009)$ analyzed the dynamics of dengue disease with the help of interrupted feeding in the role of vertical and mechanical transmission. Also, discussed a transmission model of dengue with varying vector populations and the constant human population. They also established the global stability of endemic equilibrium with the threshold condition's help to measure the population of vector control parameters. In Linda and Amy (2000), the authors compared the deterministic and stochastic SIS and SIR model in discrete time. Misra and Sharma (2013), evaluate the effect of media awareness by advertising programs on vector-borne diseases. A nonlinear mathematical model is suggested and evaluated in this article. Tewa, Dimi, and Bowong (2009) studied a dengue fever model when only one serotype existed for the dynamics and showed the global asymptotic stable condition of disease-free and endemic equilibrium. In Kar and Jana (2009), the authors proposed a vector-borne disease model with three types of controls for eradicating disease and formulated the optimal control problem. They solved the model by considering control parameters as time-dependent. Syafruddin and Noorani (2013) constructed a model and discussed the system's local and global stability behaviour. Nur, Rachman, and Abdal (2018) analyzed the ODE's SIR model system with climate factors for dengue fever transmission characteristics in closed population and explained the equilibrium's stability using Lyapunov. In Panja, Mondal, and Chattopadhyay (2018), authors identify time-dependent cost-effective control strategies to minimize dengue spread, used the optimal control theory. In this article, the authors proposed a dengue transmission model using a control variable, namely insecticide and vaccination and develop the result based on control strategy (Agusto and Khan 2018). In Bonyah et al. (2017), authors analyze an SEIR Zika epidemic model and investigate the model with basic mathematical results. Treatment is not possible if the number of infectives exceeds a certain threshold, as it is in developing countries such as India, China, Swaziland, and Brazil. In this present paper, susceptible human have categorized by considering two different classes. We have incorporated a modified model, as discussed in Syafruddin and Noorani (2013) and compared it to the single-compartment model of susceptible beings with multiple compartments. Lyapunov function has applied to this model to examine the local and global stability analysis and the basic reproduction number. The application of sensitivity analysis has been analyzing to see the manageable parameters of the model and simulated the variables.

The structure of paper is as follows: the formation and formulation of the model described in Section "Mathematical formulation". The contained covered in Sections "Positivity and boundedness of the system", and "Qualitative analysis of model" are analysis of positivity and boundedness of the system and basic 
reproduction number of the model along with estimation of the disease-free, endemic equilibrium point. Stability analysis of the model and performance of sensitivity analysis are in Sections "Stability analysis", and "Sensitivity analysis". The simulation results, as seen in Section "Numerical simulation". Finally, a detailed discussion and conclusion is presented in Section "Conclusions".

\section{Mathematical formulation}

At any time $t>0$, the total population $N_{H}$ have been sub-divided into five compartments which are: susceptible human at high risk $S_{H_{1}}$ (high risk means susceptible are supposed to contact with the disease at a higher rate. i.e. some people are not aware of the precautions, and most areas are much polluted where the virus multiplies rapidly), susceptible human at low risk $S_{H_{2}}$ (low risk means susceptible are supposed to contact with the disease at a lower rate), human exposed to dengue virus $E_{H}$, human with dengue virus $I_{H}$ and recovered human $R_{H}$. Also the total vector population $N_{v}$ have been sub-divided into three parts: susceptible vector $S_{v}$, exposed vector $E_{v}$ and infected vector $I_{v}$ also defined in Table 1. In the described part, susceptible vector are supposed to settle at a constant rate of $\pi_{v}$. When these vectors are in contact with an infected human, they acquire dengue and move to an exposed class with rate $\beta \alpha_{H} S_{v} I_{H} / N_{H}$. We consider standard incidence relation for the interaction between susceptible vector and infected humans. The moving rate $\gamma_{v}$ are the vector from exposed class to infected class. At least a vector in every class lose their life from natural reasons with rate $\mu_{v}$. In the case of vector population, the recovered class is not recognized, if the mosquito is infected from dengue virus, it remains infected throughout their entire life. The recruitment rate of individuals in the susceptible class are joined at a fixed rate of $\pi_{H}$. Newly recruited population rate $r$, join the high-risk susceptible class $S_{H_{1}}$ and rest join the low-risk susceptible class $S_{H_{2}}$. On effective contact with infected vectors, persons in classes $S_{H_{1}}$ and $S_{H_{2}}$ move to exposed class. The interaction between susceptible human and infected vectors are a type of consultative phenomenon. The persons in class $S_{\mathrm{H}_{2}}$ are assumed to contact with disease at a lower rate in comparison to persons in class $S_{H_{1}}$. The susceptible class at high risk move to exposed class with rate $\left(\beta \alpha_{v} S_{H_{1}} I_{v}\right) / N_{H}$ and low risk move to the exposed class with rate $\left(\beta \theta \alpha_{v} S_{H_{2}} I_{v}\right) / N_{H}$. The relative possibility of low-risk susceptible with respect to high-risk susceptible is denoted by $\theta$. Human of exposed class move to infected class at rate $\gamma_{H}$. Natural death rate of human is $\mu_{H}$ in each class. The schematic diagram for the transmission dynamics of dengue virus is depicted in Figure 1.

On the basis of transmission dynamics mathematical formulation for above model is:

$$
\begin{aligned}
& \frac{\mathrm{d} S_{v}}{\mathrm{~d} t}=\pi_{v}-\beta \alpha_{H} S_{v} \frac{I_{H}}{N_{H}}-\mu_{v} S_{v} \\
& \frac{\mathrm{d} E_{v}}{\mathrm{~d} t}=\beta \alpha_{H} S_{v} \frac{I_{H}}{N_{H}}-\left(\gamma_{v}+\mu_{v}\right) E_{v}
\end{aligned}
$$

Table 1: Variables used in the framework are listed.

\begin{tabular}{ll}
\hline Parameters & Significance \\
\hline$N_{H}$ & Total human population \\
$S_{H_{1}}$ & Susceptible human at high risk \\
$S_{H_{2}}$ & Susceptible human at low risk \\
$E_{H}$ & Exposed human \\
$I_{H}$ & Infected human \\
$R_{H}$ & Recovered human \\
$N_{v}$ & Total vector population \\
$S_{v}$ & Susceptible vector (mosquito) \\
$E_{v}$ & Exposed vector \\
$I_{v}$ & Infected vector \\
\hline
\end{tabular}




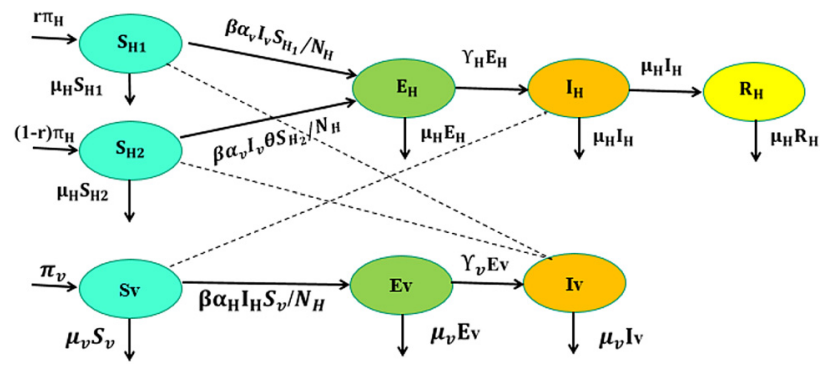

Figure 1: Compartmental model.

$$
\begin{aligned}
\frac{\mathrm{d} I_{v}}{\mathrm{~d} t} & =\gamma_{v} E_{v}-\mu_{v} I_{v} \\
\frac{\mathrm{d} S_{H_{1}}}{\mathrm{~d} t} & =r \pi_{H}-\beta \alpha_{v} I_{v} \frac{S_{H_{1}}}{N_{H}}-\mu_{H} S_{H_{1}} \\
\frac{\mathrm{d} S_{H_{2}}}{\mathrm{~d} t} & =(1-r) \pi_{H}-\beta \theta \alpha_{v} S_{H_{2}} \frac{I_{v}}{N_{H}}-\mu_{H} S_{H_{2}} \\
\frac{\mathrm{d} E_{H}}{\mathrm{~d} t} & =\beta \alpha_{v}\left(S_{H_{1}}+\theta S_{H_{2}}\right) \frac{I_{v}}{N_{H}}-\left(\gamma_{H}+\mu_{H}\right) E_{H} \\
\frac{\mathrm{d} I_{H}}{\mathrm{~d} t} & =\gamma_{H} E_{H}-\left(\mu_{H}+q\right) I_{H} \\
\frac{\mathrm{d} R_{H}}{\mathrm{~d} t} & =q I_{H}-\mu_{H} R_{H}
\end{aligned}
$$

The meaning of parameters used in (1)-(8) are listed in Tables 1 and 2.

The above system can also be written as:

$$
\begin{aligned}
\frac{\mathrm{d} S_{v}}{\mathrm{~d} t} & =\pi_{v}-d_{11} I_{H} S_{v}-\mu_{v} S_{v} \\
\frac{\mathrm{d} E_{v}}{\mathrm{~d} t} & =d_{11} I_{H} S_{v}-d_{12} E_{v} \\
\frac{\mathrm{d} I_{v}}{\mathrm{~d} t} & =\gamma_{v} E_{v}-\mu_{v} I_{v} \\
\frac{\mathrm{d} S_{H_{1}}}{\mathrm{~d} t} & =B-d_{13} I_{v} S_{H_{1}}-\mu_{H} S_{H_{1}}
\end{aligned}
$$

Table 2: Biological significance of parameters.

\begin{tabular}{ll}
\hline Parameters & Significance \\
\hline$\pi_{v}$ & Mobilization rate of susceptible vector population \\
$\beta$ & Biting rate of vector per individual \\
$\mu_{H}, \mu_{v}$ & Natural death rate of humans, vector respectively \\
$\alpha_{H}$ & Mobilization rate from infected human to suspectable vector \\
$\gamma_{v}$ & Extrinsic death rate of vector \\
$\gamma_{H}$ & Intrinsic death rate of humans \\
$\pi_{H}$ & Mobilization rate of suspectable human population \\
$q$ & Recovery rate of disease \\
$r$ & Section of first time admitted person joining the susceptible class at high risk \\
$\alpha_{v}$ & Transmission rate from infected mosquitoes to susceptible human \\
$\theta$ & Relative possibility of low risk susceptible with reference to high risk susceptible \\
\hline
\end{tabular}




$$
\begin{aligned}
\frac{\mathrm{d} S_{H_{2}}}{\mathrm{~d} t} & =A-d_{13} \theta I_{v} S_{H_{2}}-\mu_{H} S_{H_{2}} \\
\frac{\mathrm{d} E_{H}}{\mathrm{~d} t} & =d_{13} I_{v}\left(S_{H_{1}}+\theta S_{H_{2}}\right)-d_{14} E_{H} \\
\frac{\mathrm{d} I_{H}}{\mathrm{~d} t} & =\gamma_{H} E_{H}-d_{15} I_{H} \\
\frac{\mathrm{d} R_{H}}{\mathrm{~d} t} & =q I_{H}-\mu_{H} R_{H}
\end{aligned}
$$

with initial conditions, $S_{v}(0)>0, E_{v}(0) \geq 0, I_{v}(0) \geq 0, S_{H_{1}}(0)>0, S_{H_{2}}(0)>0, E_{H}(0) \geq 0, I_{H}(0) \geq 0, R_{H}(0)$ $\geq 0$, where, $d_{11}=\beta \frac{\alpha_{H}}{N_{H}}, d_{12}=\gamma_{v}+\mu_{v}, d_{13}=\beta \frac{\alpha_{v}}{N_{H}}, d_{14}=\gamma_{H}+\mu_{H}, d_{15}=\mu_{H}+q, A=(1-r) \pi_{H}, B=r \pi_{H}$.

\section{Positivity and boundedness of the system}

System (1)-(8) has positively invariant and bounded solution in the closed set.

Lemma 1. The region of existence for all solutions initiating in the positive region is recommended by set $\omega$

$$
\omega=\left\{\left(S_{H_{1}}, S_{H_{2}}, N_{v}, N_{H}\right): 0 \leq N_{v} \leq Z_{1}, 0 \leq S_{H_{1}} \leq Z_{2}, 0 \leq S_{H_{2}} \leq Z_{3}, 0 \leq N_{H} \leq Z_{4}\right.
$$

where, $Z_{1}=\max \left\{\frac{\pi_{v}}{\mu_{v}}, N_{v}(0)\right\}, \quad Z_{2}=\max \left\{\frac{r \pi_{H}}{\mu_{H}}, S_{H_{1}}(0)\right\}, \quad Z_{3}=\max \left\{\frac{(1-r) \pi_{H}}{\mu_{H}}, S_{H_{2}}(0)\right\}, \quad Z_{4}=\max \left\{\frac{\pi_{H}}{\mu_{H}}\right.$, $\left.N_{H}(0)\right\}$, which is invariant and compact with respect to the system (1)-(8).

Proof. System (1)-(8) can be written as:

$$
\begin{gathered}
\frac{\mathrm{d} Y}{\mathrm{~d} t}=C Y+D \\
Y=\left[S_{v}, E_{v}, I_{v}, S_{H_{1}}, S_{H_{2}}, E_{H}, I_{H}, R_{H}\right]^{\mathrm{T}} \\
C=\left(\begin{array}{cccccccc}
k_{1} & 0 & 0 & 0 & 0 & 0 & 0 & 0 \\
k_{2} & k_{3} & 0 & 0 & 0 & 0 & 0 & 0 \\
0 & \gamma_{v} & -\mu_{v} & 0 & 0 & 0 & 0 & 0 \\
0 & 0 & 0 & k_{4} & 0 & 0 & 0 & 0 \\
0 & 0 & 0 & 0 & k_{5} & 0 & 0 & 0 \\
0 & 0 & k_{6} & 0 & 0 & k_{7} & 0 & 0 \\
0 & 0 & 0 & 0 & 0 & \gamma_{v} & -d_{15} & 0 \\
0 & 0 & 0 & 0 & 0 & 0 & q & -\mu_{H}
\end{array}\right)
\end{gathered}
$$

where,

$$
\begin{array}{ll}
k_{1}=-\left[\mu_{v}+\beta \alpha_{H}\left(\frac{I_{H}}{N_{H}}\right)\right], & k_{2}=\beta \alpha_{H}\left(\frac{I_{H}}{N_{H}}\right), \quad k_{3}=-\left(\gamma_{v}+\mu_{v}\right) \\
k_{4}=-\left[\mu_{H}+\beta \alpha_{v}\left(\frac{I_{v}}{N_{H}}\right)\right], & k_{5}=-\left[\mu_{H}+\beta \alpha_{v} \theta\left(\frac{I_{H}}{N_{H}}\right)\right], \quad k_{6}=\beta \alpha_{v}\left(\frac{S_{H_{1}}+\theta S_{H_{2}}}{N_{H}}\right) \\
k_{7}=-\left(\gamma_{H}+\mu_{H}\right)
\end{array}
$$

The vector $D=\left[\pi_{v}, 0,0, r \pi_{H},(1-r) \pi_{H}, 0,0,0\right]^{\mathrm{T}}$ has positive nature and all of $C(Y)$ 's off-diagonal entries are non-negative. As a result, for all $Y \in R_{+}^{8}$, the matrix $C(Y)$ is Metzler (Abate, Tiwari, and Sastry 2009). Hence, all trajectories of system (1) -(8) which generate from an initial state in $Y \in R_{+}^{8}$ are bounded forever. On adding the first three equations of system (1)-(8), we get

$$
\frac{\mathrm{d} N_{v}}{\mathrm{~d} t}=\pi_{v}-\mu_{v} N_{v}
$$


Using a standard comparison theorem used in Lakshmikantham, Leela, and Martynyuk (1989) we have, $0 \leq N_{v}(t) \leq \frac{\pi_{v}}{\mu_{v}}+\left(N_{v}(0)-\frac{\pi_{v}}{\mu_{v}}\right) \mathrm{e}^{-\frac{\pi_{v}}{\mu_{v}} t}$. Thus, as $t \rightarrow \infty, 0 \leq N_{v}(t) \leq \frac{\pi_{v}}{\mu_{v}}$, for any $t>0,0 \leq N_{v}(t) \leq Z_{1}$, where $Z_{1}$ $=\max \left\{\frac{\pi_{v}}{\mu_{v}}, N_{v}(0)\right\}$.

Assume that $Z_{2}=\max \left\{\frac{r \pi_{H}}{\mu_{H}}, S_{H_{1}}(0)\right\}$, then $0 \leq S_{H_{1}} \leq Z_{2}$. Similarly, let $Z_{3}=\max \left\{\frac{(1-r) \pi_{H}}{\mu_{H}}, S_{H_{2}}(0)\right\}$, then $0 \leq S_{H_{2}} \leq Z_{3}$. By adding last five equations of system (1)-(8), we get

$$
\frac{\mathrm{d} N_{H}}{\mathrm{~d} t}=\pi_{H}-\mu_{H} N_{H}
$$

Assume that $Z_{4}=\max \left\{\frac{\pi_{H}}{\mu_{H}}, N_{H}(0)\right\}$, then $0 \leq N_{H} \leq Z_{4}$.

Therefore, all the feasible solution of system (1)-(8) are entered in the region $\omega$, which means that it is the attracting set for region $\omega$.

\section{Qualitative analysis of model}

\section{Basic reproduction number}

In this section, the basic reproduction number $\left(R_{0}\right)$ is determined by using the next-generation matrix method as in Driessch and Watmough (2002), and Ullah et al. (2020). It follows that the system will be locally asymptotically stable if $R_{0}<1$, system will be unstable if $R_{0}>1$. The process to find the basic reproduction number through Jacobian matrices $F$ and $V$ are:

$$
F=\left(\begin{array}{llll}
\frac{\partial F_{1}}{\partial E_{v}} & \frac{\partial F_{1}}{\partial I_{v}} & \frac{\partial F_{1}}{\partial E_{H}} & \frac{\partial F_{1}}{\partial I_{H}} \\
\frac{\partial F_{2}}{\partial E_{v}} & \frac{\partial F_{2}}{\partial I_{v}} & \frac{\partial F_{2}}{\partial E_{H}} & \frac{\partial F_{2}}{\partial I_{H}} \\
\frac{\partial F_{3}}{\partial E_{v}} & \frac{\partial F_{3}}{\partial I_{v}} & \frac{\partial F_{3}}{\partial E_{H}} & \frac{\partial F_{3}}{\partial I_{H}} \\
\frac{\partial F_{4}}{\partial E_{v}} & \frac{\partial F_{4}}{\partial I_{v}} & \frac{\partial F_{4}}{\partial E_{H}} & \frac{\partial F_{4}}{\partial I_{H}}
\end{array}\right) \text { and } V=\left(\begin{array}{llll}
\frac{\partial V_{1}}{\partial E_{v}} & \frac{\partial V_{1}}{\partial I_{v}} & \frac{\partial V_{1}}{\partial E_{H}} & \frac{\partial V_{1}}{\partial I_{H}} \\
\frac{\partial V_{2}}{\partial E_{v}} & \frac{\partial V_{2}}{\partial I_{v}} & \frac{\partial V_{2}}{\partial E_{H}} & \frac{\partial V_{2}}{\partial I_{H}} \\
\frac{\partial V_{3}}{\partial E_{v}} & \frac{\partial V_{3}}{\partial I_{v}} & \frac{\partial V_{3}}{\partial E_{H}} & \frac{\partial V_{3}}{\partial I_{H}} \\
\frac{\partial V_{4}}{\partial E_{v}} & \frac{\partial V_{4}}{\partial I_{v}} & \frac{\partial V_{4}}{\partial I_{H}} & \frac{\partial V_{4}}{\partial I_{H}}
\end{array}\right)
$$

where,

$$
F_{1}=d_{11} I_{H} S_{v}, \quad F_{2}=\gamma_{v} E_{v}, \quad F_{3}=d_{13} I_{v}\left(S_{H_{1}}+\theta S_{H_{2}}\right), \quad F_{4}=\gamma_{H} E_{H} .
$$

and

$$
V_{1}=d_{12} E_{v}, \quad V_{2}=\mu_{v} I_{v}, \quad V_{3}=d_{14} E_{H}, \quad V_{4}=d_{15} I_{H} .
$$

then,

$$
F=\left(\begin{array}{cccc}
0 & 0 & 0 & d_{11} S_{v} \\
\gamma_{v} & 0 & 0 & 0 \\
0 & d_{13}\left(S_{H_{1}}+\theta S_{H_{2}}\right) & 0 & 0 \\
0 & 0 & \gamma_{H} & 0
\end{array}\right) \text { and } V=\left(\begin{array}{cccc}
d_{12} & 0 & 0 & 0 \\
0 & \mu_{v} & 0 & 0 \\
0 & 0 & d_{14} & 0 \\
0 & 0 & 0 & d_{15}
\end{array}\right)
$$

then $\left(F . V^{-1}\right)$ is calculated as:

$$
F . V^{-1}=\left(\begin{array}{cccc}
0 & 0 & 0 & \frac{d_{11} \pi_{v}}{\mu_{v} d_{15}} \\
\frac{\gamma_{v}}{d_{12}} & 0 & 0 & 0 \\
0 & \frac{d_{13}(B+\theta A)}{\mu_{H} \mu_{v}} & 0 & 0 \\
0 & 0 & \frac{\gamma_{H}}{d_{14}} & 0
\end{array}\right)
$$


So, the basic reproduction number $R_{0}$ of system (9)-(16) is defined by maximum eigenvalue of the matrix $\left(F \cdot V^{-1}\right)$ and it is given by

$$
R_{0}=\sqrt{\frac{1}{\mu_{v}^{2}} \frac{d_{11} d_{13} \pi_{v} \gamma_{v} \gamma_{H}(B+A \theta)}{d_{12} d_{14} d_{15} \mu_{H}}}
$$

\section{Disease-free equilibrium}

For system (9)-(16), the disease-free equilibrium point is $W_{0}=\left(\overline{S_{v}}, \overline{E_{v}}, \overline{I_{v}}, \overline{S_{H_{1}}}, \overline{S_{H_{2}}^{-}}, \overline{E_{H}}, \overline{I_{H}}, \overline{R_{H}}\right)$. This equilibrium exists without any condition, where,

$$
\overline{S_{v}}=\frac{\pi_{v}}{\mu_{v}}, \quad \overline{E_{v}}=0, \quad \overline{I_{v}}=0, \quad \overline{S_{H_{1}}}=\frac{B}{\mu_{H}}, \quad \overline{S_{H_{2}}^{-}}=\frac{A}{\mu_{H}}, \quad \overline{E_{H}}=0, \quad \overline{I_{H}}=0, \quad \overline{R_{H}}=0 .
$$

\section{Endemic equilibrium}

For Eqs. (1)-(8) the endemic-equilibrium point is $W_{1}=\left(S_{v}^{*}, E_{v}^{*}, I_{v}^{*}, S_{H_{1}}^{*}, S_{H_{2}}^{*}, E_{H}^{*}, I_{H}^{*}, R_{H}^{*}\right)$, whose constituents are positive solutions for the equilibrium equation (23) of the system (1)-(8).

A newer variable $Z$ is defined as:

$$
Z=\beta \alpha_{v} \frac{I_{v}^{*}}{N_{H}^{*}}
$$

From the equilibrium equations (23) of the system (1)-(8), we have

$$
\begin{aligned}
& S_{v}^{*}=\frac{\pi_{v}}{\mu_{v}+\beta \alpha_{H} \frac{I_{H}^{*}}{N_{H}^{*}}}, \quad E_{v}^{*}=\frac{\beta \alpha_{H} \pi_{v} \frac{I_{H}^{*}}{N_{H}^{*}}}{\left(\gamma_{v}+\mu_{v}\right)\left[\mu_{v}+\beta \alpha_{H} \frac{I_{H}^{*}}{N_{H}^{*}}\right]}, \\
& I_{v}^{*}=\frac{\beta \alpha_{H} \gamma_{v} \pi_{v} \frac{I_{H}^{*}}{N_{H}^{*}}}{\mu_{v}\left(\gamma_{v}+\mu_{v}\right)\left[\mu_{v}+\beta \alpha_{H} \frac{I_{H}^{*}}{N_{H}^{*}}\right]}, \quad S_{H_{1}}^{*}=\frac{r \pi_{H}}{Z+\mu_{H}}, \quad S_{H_{2}}^{*}=\frac{(1-r) \pi_{H}}{\theta Z+\mu_{H}}, \\
& E_{H}^{*}=\frac{Z}{\mu_{H}+\gamma_{H}}\left[\frac{r \pi_{H}}{Z+\mu_{H}}+\theta \frac{(1-r) \pi_{H}}{\theta Z+\mu_{H}}\right], \quad I_{H}^{*}=\frac{\gamma_{H} E_{H}^{*}}{\mu_{H}+Z}, \quad R_{H}^{*}=\frac{q I_{H}^{*}}{\mu_{H}}
\end{aligned}
$$

the total human population is given by;

$$
N_{H}^{*}=\left[\frac{E_{H}^{*} \gamma_{H}}{\mu_{H}}+\frac{\pi_{H}\left(\left(Z+\mu_{H}\right)\left(Z \theta+\mu_{H}\right)+\gamma_{H}\left(Z-Z r+Z r \theta+\mu_{H}\right)\right)}{\left(Z+\mu_{H}\right)\left(\theta Z+\mu_{H}\right)\left(\mu_{H}+\gamma_{H}\right)}\right]
$$

Now using Eqs. (19)-(22) in Eq. (18), we get the following quadratic equation in $Z$ :

$$
B_{3} Z^{3}+B_{2} Z^{2}+B_{1} Z+B_{0}=0
$$

where,

$$
\begin{aligned}
B_{3}= & \mu_{H}^{2}\left(\left(1+4 \theta+(\theta)^{2}\right) \pi_{H}\left(q+\mu_{H}\right)^{2}\left(\mu_{H}+\gamma_{H}\right)^{2} \mu_{v}+\beta^{2} \theta \pi_{v} \alpha_{H} \alpha_{v} \gamma_{H} \gamma_{v} \mu_{H}\left(\gamma_{v}+\mu_{v}\right)(2 \beta(r(\theta-1)\right. \\
& \left.-\theta) \alpha_{H} \gamma_{H} \mu_{H}+(-1+r(\theta-1)-2 \theta)\left(q+\mu_{H}\right)\left(\gamma_{H}+\mu_{H}\right) \mu_{v}\right) \\
B_{2}= & \mu_{H}^{3}\left(2(1+\theta) \pi_{H}\left(q+\mu_{H}\right)^{2}\left(\gamma_{H}+\mu_{H}\right)^{2} \mu_{v}-\beta^{2} \pi_{v} \alpha_{H} \alpha_{v} \mu_{H}\left(\gamma_{v}+\mu_{v}\right)\left(\beta(r+\theta-r \theta)^{2} \alpha_{H} \gamma_{H} \mu_{H}\right.\right. \\
& \left.\left.-\left(-\theta(2+\theta)+r\left(-1+\theta^{2}\right)\right)\left(q+\mu_{H}\right)\left(\gamma_{H}+\mu_{H}\right) \mu_{v}\right)\right) \\
B_{1}= & \mu_{H}^{4}\left(q+\mu_{H}\right)^{2}\left(\gamma_{H}+\mu_{H}\right)^{2} \mu_{v}^{2}\left(\gamma_{v}+\mu_{v}\right) \pi_{H}\left(1-R_{0}^{2}\right) \\
B_{0}= & 0 .
\end{aligned}
$$

On applying Descartes' rule of sign, we discussed the various chances for positive roots of Eq. (23) in Table 3. Here $B_{0}=0$, therefore it has no effect on evaluation of roots as in Driessche (2017) and Ghosh, Tiwari, and Chttopadhyay (2019). 
Table 3: For $R_{0}<1$ and $R_{0}>1$ number of possible positive roots of Eq. (23).

\begin{tabular}{lrlrr}
\hline S.N. & $\boldsymbol{B}_{\mathbf{3}} \boldsymbol{B}_{\mathbf{2}} \boldsymbol{B}_{\mathbf{1}} \boldsymbol{B}_{\mathbf{0}}$ & $\boldsymbol{R}_{\mathbf{0}}$ & Change of sign & Possibility of positive root \\
\hline 1 & +++0 & $R_{0}<1$ & 0 & 0 \\
& ++-0 & $R_{0}>1$ & 1 & 1 \\
2 & +-+0 & $R_{0}<1$ & 2 & 0,2 \\
& +--0 & $R_{0}>1$ & 1 & 1 \\
3 & -++0 & $R_{0}<1$ & 1 & 1 \\
& -+-0 & $R_{0}>1$ & 2 & 1,2 \\
4 & --+0 & $R_{0}<1$ & 1 & 1 \\
& ---0 & $R_{0}>1$ & 0 & 0 \\
\hline
\end{tabular}

\section{Stability analysis}

In this section, the stability analysis of disease-free and endemic equilibrium is carried out. The basic reproduction number $\left(R_{0}\right)$ is used to discuss both the equilibrium points regarding local stability and global stability. Stability analysis can perform, using the sign of eigenvalues of the Jacobian matrix's characteristic equation as in Esteva and Vargas (1998) and Lakshmikantham, Leela, and Martynyuk (1989).

\section{Local stability around equilibrium point}

Now in this section, we are going to analyze the stability of two equilibrium points $W_{0}$ and $W_{1}$. The outcome of stability analysis of these equilibria are shown in the following two theorems.

Theorem 1. For $R_{0}<1$ the disease-free equilibrium $\left(W_{0}\right)$ is locally asymptotically stable and unstable if $R_{0}>1$.

Proof. The Jacobian matrix $W_{01}$ for the model system (9)-(16) corresponding to equilibrium $W_{0}$ is given by

$$
W_{01}=\left(\begin{array}{cccccccc}
-\mu_{v} & 0 & 0 & 0 & 0 & 0 & \frac{-d_{11} \pi_{v}}{\mu_{v}} & 0 \\
0 & -d_{12} & 0 & 0 & 0 & 0 & \frac{d_{11} \pi_{v}}{\mu_{v}} & 0 \\
0 & \gamma_{v} & -\mu_{v} & 0 & 0 & 0 & 0 & 0 \\
0 & 0 & \frac{-d_{13} B}{\mu_{H}} & -\mu_{H} & 0 & 0 & 0 & 0 \\
0 & 0 & \frac{-d_{13} A \theta}{\mu_{H}} & 0 & -\mu_{H} & 0 & 0 & 0 \\
0 & 0 & \frac{d_{13}(B+\theta A)}{\mu_{H}} & 0 & 0 & -d_{14} & 0 & 0 \\
0 & 0 & 0 & 0 & 0 & \gamma_{H} & -d_{15} & 0 \\
0 & 0 & 0 & 0 & 0 & 0 & q & -\mu_{H}
\end{array}\right)
$$

The four eigen values of $W_{01}$ at disease-free equilibrium are $-\mu_{v},-\mu_{H}$ (multiplicity 3 ) and the remaining four eigenvalues are given by the following biquadratic polynomial

$$
\lambda^{4}+v_{1} \lambda^{3}+v_{2} \lambda^{2}+v_{3} \lambda+v_{4}=0
$$

where,

$$
\begin{aligned}
& v_{1}=d_{12}+d_{14}+d_{15}+\mu_{v} \\
& v_{2}=d_{12} d_{14}+d_{12} d_{15}+d_{14} d_{15}+d_{12} \mu_{v}+d_{14} \mu_{v}+d_{15} \mu_{v} \\
& v_{3}=d_{12} d_{14} d_{15}+d_{12} d_{14} \mu_{v}+d_{12} d_{15} \mu_{v}+d_{14} d_{15} \mu_{v} \\
& v_{4}=d_{12} d_{14} d_{15} \mu_{v}\left(1-R_{0}^{2}\right) .
\end{aligned}
$$


Here, $v_{1}>0, v_{1} v_{2}-v_{3}>0, v_{1} v_{2} v_{3}-v_{3}^{2}-v_{4} v_{1}^{2}>0$.

So, if $R_{0}<1$, all of the above conditions are fulfilled. Hence by Routh-Hurwitz criterion the disease-free equilibrium point $W_{0}$ is locally asymptotically stable otherwise, it is unstable.

Theorem 2. The endemic equilibrium $W_{1}$ for the system (9)-(16) is locally asymptotically stable under some conditions stated in the proof of this theorem.

Proof. The Jacobian matrix $W_{10}$ for the model system (9)-(16) corresponding to equilibrium $W_{1}$ is given by

$$
W_{10}=\left(\begin{array}{cccccccc}
-A_{1} & 0 & 0 & 0 & 0 & 0 & -d_{11} S_{v}^{*} & 0 \\
d_{11} I_{H}^{*} & -d_{12} & 0 & 0 & 0 & 0 & d_{11} S_{v}^{*} & 0 \\
0 & \gamma_{v} & -\mu_{v} & 0 & 0 & 0 & 0 & 0 \\
0 & 0 & -A_{2} & -A_{3} & 0 & 0 & 0 & 0 \\
0 & 0 & -A_{4} & 0 & -A_{5} & 0 & 0 & 0 \\
0 & 0 & A_{6} & A_{7} & A_{8} & -d_{14} & 0 & 0 \\
0 & 0 & 0 & 0 & 0 & \gamma_{H} & -d_{15} & 0 \\
0 & 0 & 0 & 0 & 0 & 0 & q & -\mu_{H}
\end{array}\right)
$$

where, $A_{1}=d_{11} I_{H}^{*}+\mu_{v}, A_{2}=d_{13} S_{H_{1}}^{*}, A_{3}=d_{13} I_{v}^{*}+\mu_{H}, A_{4}=d_{13} \theta S_{H_{2}}^{*}, A_{5}=d_{13} \theta I_{v}^{*}+\mu_{H}, A_{6}=d_{13}\left(S_{H_{1}}^{*}+\theta S_{H_{2}}^{*}\right)$, $A_{7}=d_{13} I_{v}^{*}, A_{8}=d_{13} \theta I_{v}^{*}$

Four eigenvalues of the above matrix are $-A_{1},-\mu_{H},-A_{3},-A_{5}$ and the other roots are given with the help of biquadratic polynomial

$$
\lambda^{4}+w_{1} \lambda^{3}+w_{2} \lambda^{2}+w_{3} \lambda+w_{4}=0
$$

where,

$$
\begin{aligned}
& w_{1}=d_{12}+d_{14}+d_{15}+\mu_{v} \\
& w_{2}=d_{15} \mu_{v}+d_{14}\left(d_{15}+\mu_{v}\right)+d_{12}\left(d_{14}+d_{15}+\mu_{v}\right)-A_{6} d_{11} \gamma_{H} \gamma_{v} S_{v}^{*} \\
& w_{3}=d_{12} d_{14} d_{15}+d_{14} d_{15} \mu_{v}+d_{12}\left(d_{14}+d_{15}\right) \mu_{v}+\left(-\left(A_{3}+A_{5}\right) A_{6}+A_{2} A_{7}+A_{4} A_{8}\right) d_{11} \gamma_{H} \gamma_{v} S_{v}^{*} \\
& w_{4}=d_{12} d_{14} d_{15} \mu_{v}\left(1-A_{3} A_{5} R_{0}^{2}\right)+\left(A_{2} A_{5} A_{7}+A_{3} A_{4} A_{8}\right) d_{11} \gamma_{H} \gamma_{v} S_{v}^{*} .
\end{aligned}
$$

Here, $w_{1}>0, w_{1} w_{2}-w_{3}>0, w_{1} w_{2} w_{3}-w_{3}^{2}-w_{4} w_{1}^{2}>0$.

For this, if $w_{4}>0$ and $R_{0}>\frac{1}{A_{3} A_{5}}$, the roots of Eq. (25) will have negative real parts. Therefore, Routh-Hurwitz criterion satisfied and the system is locally asymptotically stable for $W_{1}$.

\section{Global stability around equilibrium point}

Here, we are going to analyze the stability of two equilibrium points $W_{0}$ and $W_{1}$. The outcomes of stability analysis of these equilibrium points are shown in the following two theorems.

Theorem 1. If $R_{0} \leq 1$, the disease-free equillibrium $\left(W_{0}\right)$ is globally asymptotically stable on $\omega$ with assumption

$$
\begin{array}{r}
d_{13}\left(\bar{S}_{H_{1}}+\theta \bar{S}_{H_{2}}\right)=\mu_{v} \\
d_{11} \bar{S}_{v}=q+\mu_{H} .
\end{array}
$$

Proof. Consider the Lyapunov function below:

$$
G(t)=\left(S_{v}-\bar{S}_{v} \ln S_{v}\right)+E_{v}+I_{v}+\left(S_{H_{1}}-\bar{S}_{H_{1}} \ln S_{H_{1}}\right)+\left(S_{H_{2}}-\bar{S}_{H_{2}} \ln S_{H_{2}}\right)+E_{H}+I_{H}+R_{H}
$$


Differentiating with respect to time $t$, get:

$$
\begin{aligned}
G^{\prime}(t)= & S_{v}^{\prime}\left(1-\frac{\bar{S}_{v}}{S_{v}}\right)+E_{v}^{\prime}+I_{v}^{\prime}+S_{H_{1}}^{\prime}\left(1-\frac{\bar{S}_{H_{1}}}{S_{H_{1}}}\right)+S_{H_{2}}^{\prime}\left(1-\frac{\bar{S}_{H_{2}}}{S_{H_{2}}}\right)+E_{H}^{\prime}+I_{H}^{\prime}+R_{H}^{\prime} \\
G^{\prime}(t)= & \left(\pi_{v}-d_{11} I_{H} S_{v}-\mu_{v} S_{v}\right)\left(1-\frac{\bar{S}_{v}}{S_{v}}\right)+d_{11} I_{H} S_{v}-d_{12} E_{v}+\gamma_{v} E_{v}-\mu_{v} I_{v} \\
& +\left(B-d_{13} I_{v} S_{H_{1}}-\mu_{H} S_{H_{1}}\right)\left(1-\frac{\bar{S}_{H_{1}}}{S_{H_{1}}}\right)+\left(A-d_{13} \theta I_{v} S_{H_{2}}-\mu_{H} S_{H_{2}}\right)\left(1-\frac{\bar{S}_{H_{2}}}{S_{H_{2}}}\right) \\
& +d_{13} I_{v}\left(S_{H_{1}}+\theta S_{H_{2}}\right)-d_{14} E_{H}+\gamma_{H} E_{H}-d_{15} I_{H}+q I_{H}-\mu_{H} R_{H}
\end{aligned}
$$

On solving further get:

$$
\begin{aligned}
= & \pi_{v}\left(1-\frac{\bar{S}_{v}}{S_{v}}\right)+\mu_{v} \bar{S}_{v}\left(1-\frac{S_{v}}{\bar{S}_{v}}\right)+I_{v}\left(d_{13}\left(\bar{S}_{H_{1}}+\theta \bar{S}_{H_{2}}\right)-\mu_{v}\right)-\mu_{v} E_{v}+B\left(1-\frac{\bar{S}_{H_{1}}}{S_{H_{1}}}\right) \\
& +A\left(1-\frac{\bar{S}_{H_{2}}}{S_{H_{2}}}\right)+\mu_{H} \bar{S}_{H_{1}}\left(1-\frac{S_{H_{1}}}{\bar{S}_{H_{1}}}\right)+\mu_{H} \bar{S}_{H_{2}}\left(1-\frac{S_{H_{2}}}{\bar{S}_{H_{2}}}\right)+I_{H}\left(d_{11} \bar{S}_{v}-\left(q+\mu_{H}\right)\right)-\mu_{H} E_{H} \\
& -\mu_{H} R_{H}
\end{aligned}
$$

Using the conditions (26) and (27), also we have $\bar{S}_{v}=\frac{\pi_{v}}{\mu_{v}}, \bar{S}_{H_{1}}=\frac{B}{\mu_{H}}$ and $S_{H_{2}}=\frac{A}{\mu_{H}}$, the above equation can be rewritten as:

$$
\begin{aligned}
G^{\prime}(t)= & \pi_{v}\left(2-\frac{\bar{S}_{v}}{S_{v}}-\frac{S_{v}}{\bar{S}_{v}}\right)+B\left(2-\frac{\bar{S}_{H_{1}}}{S_{H_{1}}}-\frac{S_{H_{1}}}{\bar{S}_{H_{1}}}\right)+A\left(2-\frac{\bar{S}_{H_{2}}}{S_{H_{2}}}-\frac{S_{H_{2}}}{\bar{S}_{H_{2}}}\right)-\mu_{H} E_{H}-\mu_{v} E_{v} \\
& -\mu_{H} R_{H} \\
= & -\pi_{v} \frac{\left(S_{v}-\bar{S}_{v}\right)^{2}}{S_{v} \bar{S}_{v}}-B \frac{\left(S_{H_{1}}-\bar{S}_{H_{1}}\right)^{2}}{S_{H_{1}} \bar{S}_{H_{1}}}-A \frac{\left(S_{H_{2}}-\bar{S}_{H_{2}}\right)^{2}}{S_{H_{2}} \bar{S}_{H_{2}}}-\mu_{H} E_{H}-\mu_{v} E_{v}-\mu_{H} R_{H}
\end{aligned}
$$

Hence the conditions (26) and (27) ensures that $G^{\prime}(t) \leq 0$. By using Lasalle's extension to Lyapunov's method (Bonyah et al. 2017; Ullah, Khan, and Farooq 2018a), the limit set of each solution is contained in the largest invariant set for which $S_{H_{1}}=\bar{S}_{H_{1}}, S_{H_{2}}=\bar{S}_{H_{2}}, R_{H}=0$ and $S_{v}=\bar{S}_{v}$ is the singleton set $\left\{W_{0}\right\}$. This means, the disease-free equilibrium $\left(W_{0}\right)$ is globally asymptotically stable on $\omega$. This concludes the proof.

Theorem 2. The endemic equilibrium $\left(W_{1}\right)$ is globally asymptotically stable in $\omega$ provided the following inequalities hold

$$
\begin{array}{r}
\operatorname{Max}\left\{\left(\frac{d_{13} \theta \pi_{H}}{Z+\mu_{H}}\right)^{2},\left(\frac{d_{13}(1-r) \pi_{H} \theta}{\theta Z+\mu_{H}}\right)^{2}\right\}<\mu_{H} \mu_{v} \\
\operatorname{Max}\left\{d_{12} Z_{1}^{2}, d_{13} Z_{1} \theta, \frac{4}{\mu_{v}}\left(d_{13}\left(\frac{r \pi_{H}}{Z+\mu_{H}}+\frac{\theta(1-r) \pi_{H}}{\theta Z+\mu_{H}}\right)\right)^{2}\right\}<d_{14} \\
\operatorname{Max}\left\{\frac{4\left(\gamma_{H}\right)^{2}}{d_{14}}, \frac{q^{2}}{\mu_{H}}, d_{11} Z_{1}^{2}\right\}<d_{15} \\
\operatorname{Max}\left\{\frac{3}{4} \frac{\left(d_{11} Z_{4}\right)^{2}}{\mu_{v}}, \frac{3\left(d_{11} Z_{1}\right)^{2}}{d_{15}}, \frac{3 \gamma_{v}^{2}}{\mu_{v}}\right\}<d_{12}
\end{array}
$$


Proof. Consider the following positive definite function, to study the global stability behaviour of the endemic equilibrium $\left(W_{1}\right)$ :

$$
\begin{aligned}
V(t)= & \frac{1}{2}\left(S_{H_{1}}-S_{H_{2}}^{*}\right)^{2}+\frac{1}{2}\left(S_{H_{2}}-S_{H_{2}}^{*}\right)^{2}+\frac{1}{2}\left(E_{H}-E_{H}^{*}\right)^{2}+\frac{1}{2}\left(I_{H}-I_{H}^{*}\right)^{2} \\
& +\frac{1}{2}\left(R_{H}-R_{H}^{*}\right)^{2}+\frac{1}{2}\left(S_{v}-S_{v}^{*}\right)^{2}+\frac{1}{2}\left(E_{v}-E_{v}^{*}\right)^{2}+\frac{1}{2}\left(I_{v}-I_{v}^{*}\right)^{2}
\end{aligned}
$$

Differentiating with respect to time $t$,

$$
\begin{aligned}
V^{\prime}(t)= & S_{H_{1}}^{\prime}\left(S_{H_{1}}-S_{H_{1}}^{*}\right)+S_{H_{2}}^{\prime}\left(S_{H_{2}}-S_{H_{2}}^{*}\right)+E_{H}^{\prime}\left(E_{H}-E_{H}^{*}\right)+I_{H}^{\prime}\left(I_{H}-I_{H}^{*}\right) \\
& +R_{H}^{\prime}\left(R_{H}-R_{H}^{*}\right)+S_{v}^{\prime}\left(S_{v}-S_{v}^{*}\right)+E_{v}^{\prime}\left(E_{v}-E_{v}^{*}\right)+I_{v}^{\prime}\left(I_{v}-I_{v}^{*}\right)
\end{aligned}
$$

With the help of Eqs. (9)-(16) on rearranging the terms we get:

$$
\begin{aligned}
V^{\prime}(t)= & -\mu_{H}\left(S_{H_{1}}^{*}-S_{H_{1}}\right)^{2}+\frac{d_{13} r \pi_{H}}{Z+\mu_{H}}\left(I_{v}-I_{v}^{*}\right)\left(S_{H_{1}}^{*}-S_{H_{1}}\right)-\frac{\mu_{v}}{4}\left(I_{v}-I_{v}^{*}\right)^{2} \\
& -d_{13} Z_{1}\left(S_{H_{1}}-S_{H_{1}}^{*}\right)^{2}+d_{13} Z_{1}\left(S_{H_{1}}-S_{H_{1}}^{*}\right)\left(E_{H}-E_{H}^{*}\right)-\frac{d_{14}}{4}\left(E_{H}-E_{H}^{*}\right)^{2} \\
& -\mu_{H}\left(S_{H_{2}}^{*}-S_{H_{2}}\right)+\frac{d_{13}(1-r) \pi_{H} \theta}{\theta Z+\mu_{H}}\left(I_{v}-I_{v}^{*}\right)\left(S_{H_{2}}^{*}-S_{H_{2}}\right)-\frac{\mu_{v}}{4}\left(I_{v}-I_{v}^{*}\right)^{2} \\
& -d_{13} \theta Z_{1}\left(S_{H_{2}}-S_{H_{2}}^{*}\right)^{2}+d_{13} \theta Z_{1}\left(S_{H_{2}}-S_{H_{2}}^{*}\right)\left(E_{H}-E_{H}^{*}\right)-\frac{d_{14}}{4}\left(E_{H}-E_{H}^{*}\right)^{2} \\
& -\frac{d_{14}}{4}\left(E_{H}-E_{H}^{*}\right)^{2}+d_{13}\left(\frac{r \pi_{H}}{Z+\mu_{H}}+\frac{\theta(1-r) \pi_{H}}{\theta Z+\mu_{H}}\right)\left(I_{v}-I_{v}^{*}\right)\left(E_{H}-E_{H}^{*}\right) \\
& -\frac{\mu_{v}}{4}\left(I_{v}-I_{v}^{*}\right)^{2}-\frac{d_{15}}{4}\left(I_{H}-I_{H}^{*}\right)^{2}+\gamma_{H}\left(E_{H}-E_{H}^{*}\right)\left(I_{H}-I_{H}^{*}\right)-\frac{d_{14}}{4}\left(E_{H}-E_{H}^{*}\right)^{2} \\
& -\mu_{H}\left(R_{H}-R_{H}^{*}\right)^{2}+q\left(R_{H}-R_{H}^{*}\right)\left(I_{H}-I_{H}^{*}\right)-\frac{d_{15}}{4}\left(I_{H}-I_{H}^{*}\right)^{2} \\
& -d_{11}\left(\frac{\gamma_{H}}{\mu_{H}+q}\left(\frac{Z}{\mu_{H}+\gamma_{H}}\left(\frac{r \pi_{H}}{Z+\mu_{H}}+\frac{\theta(1-r) \pi_{H}}{\theta Z+\mu_{H}}\right)\right)\right)\left(S_{v}-S_{v}^{*}\right)^{2} \\
& +d_{11} Z_{1}\left(I_{H}-I_{H}^{*}\right)\left(S_{v}-S_{v}^{*}\right)-\frac{d_{15}}{4}\left(I_{H}-I_{H}^{*}\right)^{2}-\mu_{v}\left(S_{v}-S_{v}^{*}\right)^{2} \\
& +d_{11} Z_{4}\left(S_{v}-S_{v}^{*}\right)\left(E_{v}-E_{v}^{*}\right)-\frac{d_{12}}{3}\left(E_{v}-E_{v}^{*}\right)^{2}-\frac{d_{12}}{3}\left(E_{v}-E_{v}^{*}\right)^{2} \\
& +d_{11} Z_{1}\left(I_{H}-I_{H}^{*}\right)\left(E_{v}-E_{v}^{*}\right)-\frac{d_{15}}{4}\left(I_{H}-I_{H}^{*}\right)^{2}-\frac{d_{12}}{3}\left(E_{v}-E_{v}^{*}\right)^{2} \\
& +\gamma_{v}\left(I_{v}-I_{v}^{*}\right)\left(E_{v}-E_{v}^{*}\right)-\frac{\mu_{v}}{4}\left(I_{v}-I_{v}^{*}\right)^{2} .
\end{aligned}
$$

For system (9)-(16) inside the region of attraction $\omega, V^{\prime}(t)$ can be turned negative definite if conditions (28)-(31) hold as in Baniya and Keval (2020).

\section{Sensitivity analysis}

Here, we deal with the sensitivity analysis of the model to see the effect of manageable parameters of the model on the basic reproduction number $\left(R_{0}\right)$ as in Zheng and Nie (2018) and Agusto and Khan (2018). The basic reproduction number behave like an important epidemiological parameter, and sensitivity analysis gives us outputs of the model, divided for quantitative values through variations in different parameter values. Here, 
a specified space of parameter examines the response model from parameter variation to output variables. The sensitivity index for a variable $\gamma$, which depends differentially on a parameter $\sigma$, is defined as:

$$
Z_{\gamma}^{\sigma}=\frac{\partial \gamma}{\partial \sigma} \times \frac{\sigma}{\gamma}
$$

We measure elasticities $\left(Z_{\gamma}^{\sigma}\right)$ to see the results of relative changes to the parameter $R_{0}$. Elasticity is the proportional solution to the proportional perambulation.

$$
\begin{gathered}
Z_{R_{0}}^{\beta}=\frac{\partial R_{0}}{\partial \beta} \times \frac{\beta}{R_{0}}=\frac{(B+A \theta) \beta \pi_{v} \alpha_{H} \alpha_{v} \gamma_{H} \gamma_{v}}{\mu_{v}^{2} \mu_{H} N_{H}^{2}\left(\mu_{H}+q\right)\left(\mu_{H}+\gamma_{H}\right)\left(\mu_{v}+\gamma_{v}\right) \sqrt{G}} \times \frac{\beta}{R_{0}}>0 . \\
Z_{R_{0}}^{\alpha_{H}}=\frac{\partial R_{0}}{\partial \alpha_{H}} \times \frac{\alpha_{H}}{R_{0}}=\frac{(B+A \theta) \beta^{2} \pi_{v} \alpha_{v} \gamma_{H} \gamma_{v}}{2 \mu_{v}^{2} \mu_{H} N_{H}^{2}\left(\mu_{H}+q\right)\left(\mu_{H}+\gamma_{H}\right)\left(\mu_{v}+\gamma_{v}\right) \sqrt{G}} \times \frac{\alpha_{H}}{R_{0}}>0 . \\
Z_{R_{0}}^{\alpha_{v}}=\frac{\partial R_{0}}{\partial \alpha_{v}} \times \frac{\alpha_{v}}{R_{0}}=\frac{(B+A \theta) \beta^{2} \pi_{v} \alpha_{H} \gamma_{H} \gamma_{v}}{2 \mu_{v}^{2} \mu_{H} N_{H}^{2}\left(\mu_{H}+q\right)\left(\mu_{H}+\gamma_{H}\right)\left(\mu_{v}+\gamma_{v}\right) \sqrt{G}} \times \frac{\alpha_{v}}{R_{0}}>0 . \\
Z_{R_{0}}^{\gamma_{v}}=\frac{\partial R_{0}}{\partial \gamma_{v}} \times \frac{\gamma_{v}}{R_{0}}=\frac{-\frac{R_{0}^{2}}{\left(\mu_{v}+\gamma_{v}\right)}+\frac{R_{0}^{2}}{2 \sqrt{G}} \times \frac{\gamma_{v}}{R_{0}}<0 .}{Z_{R_{0}}^{\gamma_{H}}}=\frac{\partial R_{0}}{\partial \gamma_{H}} \times \frac{\gamma_{H}}{R_{0}}=\frac{-\frac{R_{0}^{2}}{\left(\mu_{H}+\gamma_{H}\right)}+\frac{R_{0}^{2}}{\gamma_{H}}}{2 \sqrt{G}} \times \frac{\gamma_{H}}{R_{0}}<0 . \\
Z_{R_{0}}^{\mu_{v}}=\frac{\partial R_{0}}{\partial \mu_{v}} \times \frac{\mu_{v}}{R_{0}}=\frac{-\frac{R_{0}^{2}}{\left(\mu_{v}+\gamma_{v}\right)}-\frac{2 R_{0}^{2}}{\mu_{v}}}{2 \sqrt{G}} \times \frac{\mu_{v}}{R_{0}}<0 . \\
Z_{R_{0}}^{\mu_{H}}=\frac{\partial R_{0}}{\partial \mu_{H}} \times \frac{\mu_{H}}{R_{0}}=\frac{\left(\frac{R_{0}^{2}}{\left(\mu_{H}+\gamma_{H}\right)}+\frac{R_{0}^{2}}{\left(\mu_{H}+q\right)}+\frac{R_{0}^{2}}{\mu_{H}}\right)}{2 \sqrt{G}} \times \frac{\mu_{H}}{R_{0}}<0 . \\
Z_{R_{0}}^{q}=\frac{\partial R_{0}}{\partial \pi_{v}} \times \frac{\pi_{v}}{R_{0}}=\frac{(B+A \theta) \beta^{2} \alpha_{H} \alpha_{v} \gamma_{H} \gamma_{v}}{2 q} \times \frac{q}{R_{0}}=\frac{\pi_{v}^{2} \mu_{H} N_{H}^{2}\left(\mu_{H}+q\right)\left(\mu_{H}+\gamma_{H}\right)\left(\mu_{v}+\gamma_{v}\right) \sqrt{G}}{2 \mu_{v}^{2} \mu_{H} N_{H}^{2}\left(\mu_{H}+q\right)^{2}\left(\mu_{H}+\gamma_{H}\right)\left(\gamma_{v}+\mu_{v}\right) \sqrt{G}} \times \frac{(B}{R_{0}}<0 .
\end{gathered}
$$

where, $G=\frac{(B+A \theta) \beta^{2} \pi_{v} \alpha_{H} \alpha_{v} \gamma_{H} \gamma_{v}}{\mu_{v}^{2} \mu_{H} N_{H}^{2}\left(\mu_{H}+q\right)\left(\mu_{H}+\gamma_{H}\right)\left(\mu_{v}+\gamma_{v}\right)}$

From the above expression, we see that $Z_{R_{0}}^{\alpha_{H}}=Z_{R_{0}}^{\alpha_{v}}=Z_{R_{0}}^{\pi_{v}}$ which implies small changes in any of these parameters $\alpha_{H}, \alpha_{v}$ and $\pi$ provides a significant influence. In Figures 2-10, we have demonstrated the effect of these parameters on basic reproduction number. From Figure 2, we can see that the parameters $\alpha_{H}$ and $\alpha_{v}$ have equal impact on $R_{0}$ which shows that controlling the route of transmission of this disease is very important if we plan to restrict the transmission potential. From Figures $3-5$, it is clear that the parameter $\beta$ which is biting rate has more influence on the basic reproduction number $R_{0}$ as compared to the transmission probabilities $\alpha_{v}, \alpha_{H}$ and mosquito death rate $\mu_{v}$. Although the human to human transmission of this disease is deficient, our proposed model suggests that even a minor increase in the parameter $\beta$ corresponding to

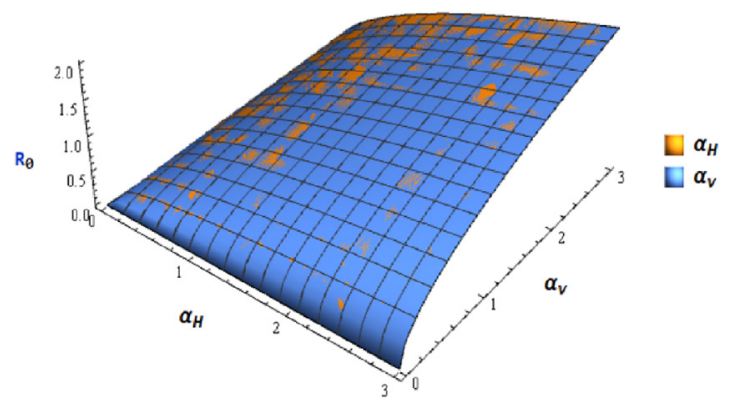

Figure 2: Influence of $\alpha_{H}$ and $\alpha_{v}$ on $R_{0}$. 

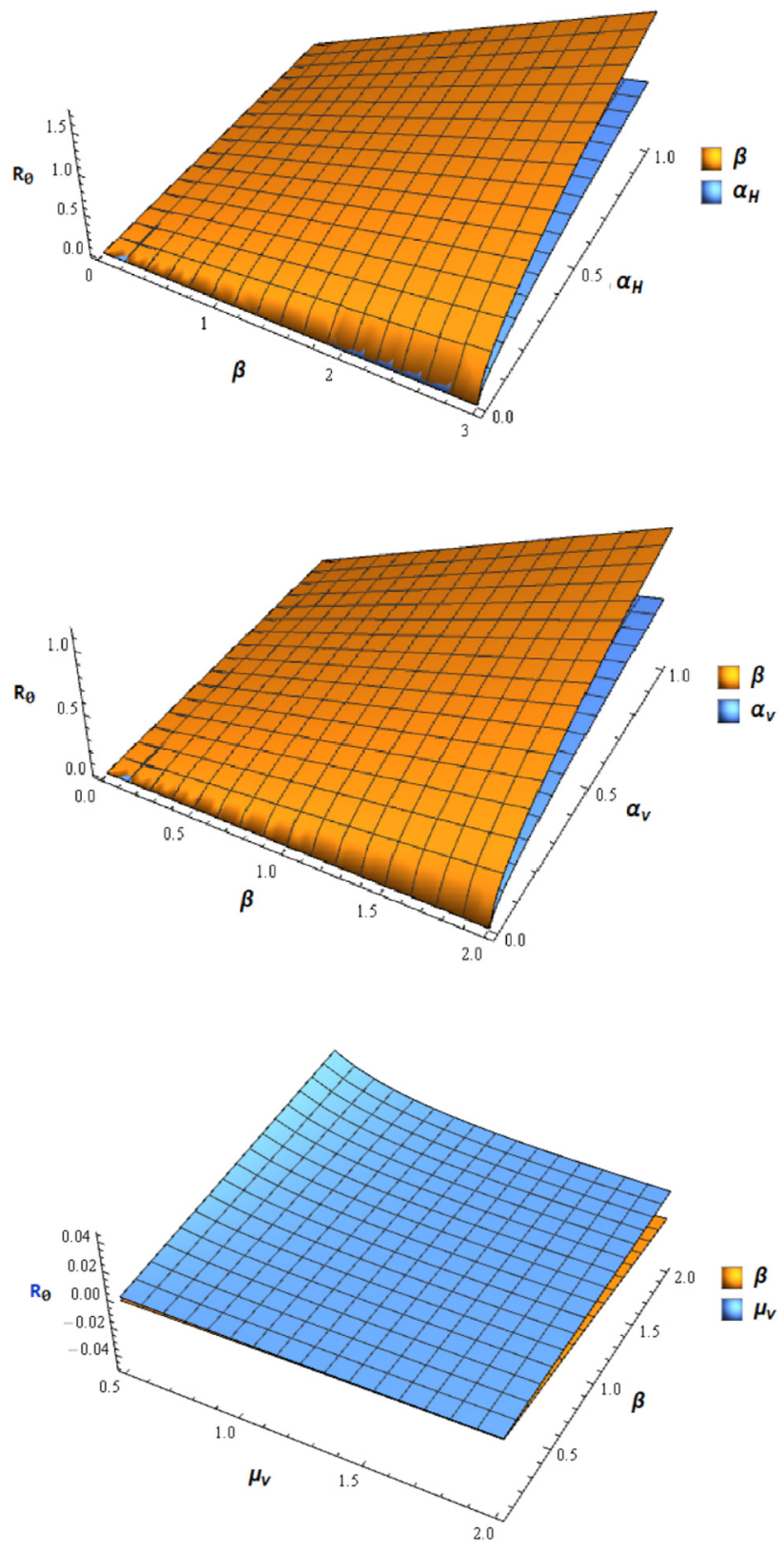

Figure 3: Influence of $\alpha_{H}$ and $\beta$ on $R_{0}$.

Figure 5: Influence of $\beta$ and $\mu_{v}$ on $R_{0}$.

transmission of dengue virus can lead to a great increase in the $R_{0}$. In Figure 6 demonstrates the fact that the impact of $\gamma_{H}$ on $R_{0}$ is much higher than the impact of $\gamma_{v}$ on $R_{0}$. Another fact clearly shown in Figures 7-9, $\beta$ is more affected by numerous biological $\left(\gamma_{v}\right)$, socio-behavioural $\left(\pi_{v}\right)$, and environmental factors $(q)$ and that govern pathogen transmission. Global sensitivity is performed in Figure 10 to determine the model's valuable parameter. Also, use the combination of Latin hypercube sampling (LHS) in conjunction with partial rank correlation coefficient (PRCC) to see the impact of manageable parameters of the model on basic reproduction number $\left(R_{0}\right)$ (Altaf Khana, Ullah, and Farooqb 2018; Khan and Atangana 2020). The parameters used in the present analysis are given in Table 4. The most sensitive parameter is $\mu_{v}$ with a low PRCC value. From the above figures, we can conclude that the control policies should reduce the transmission rates, mobilization rate of vector, biting rate, and human death rate. We can conclude that controlling this disease's transmission route is very important if we plan to restrict its transmission. Therefore, both the cases are closely related to control dengue. 


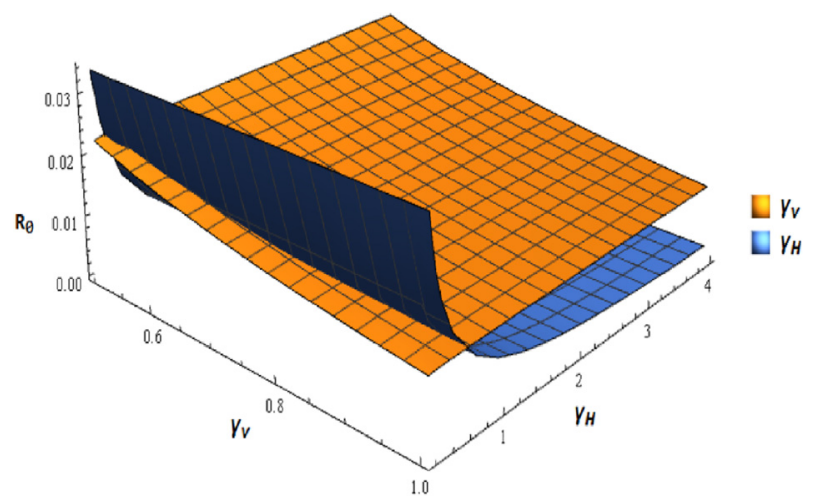

Figure 6: Influence of $\gamma_{H}$ and $\gamma_{v}$ on $R_{0}$.

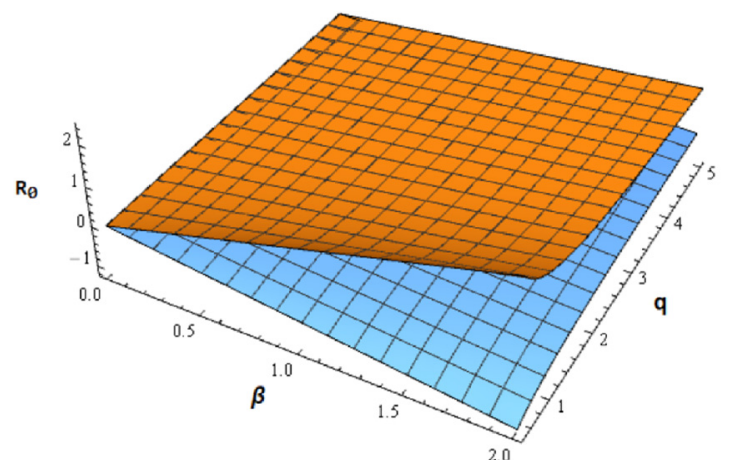

$\square \beta$
$\square q$

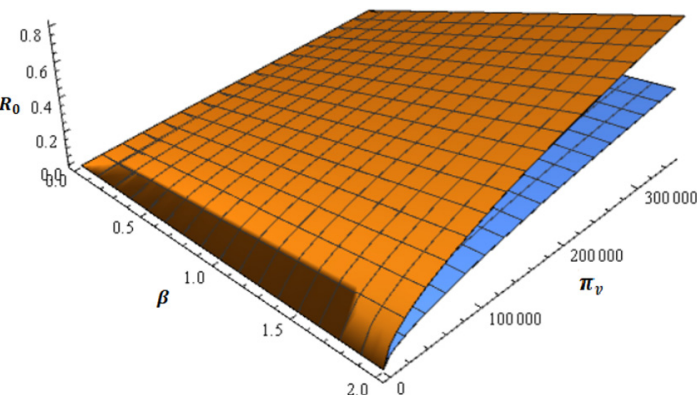

$\square \beta$

Figure 7: Influence of $\beta$ and $q$ on $R_{0}$.

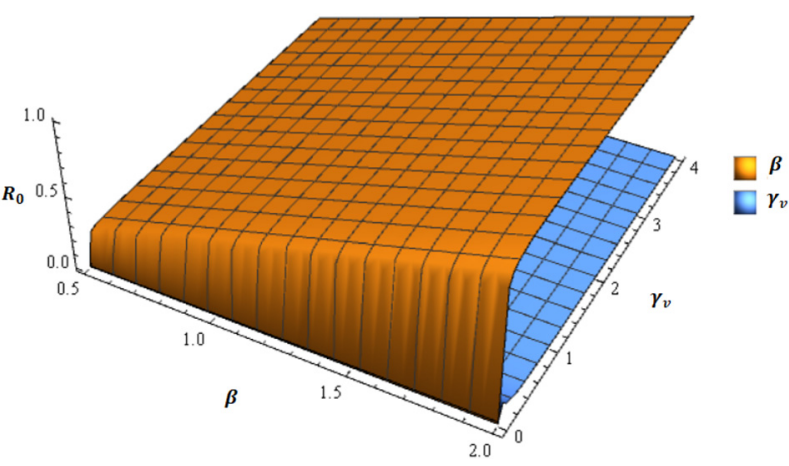

Figure 8: Influence of $\beta$ and $\pi_{v}$ on $R_{0}$.

Figure 9: Influence of $\beta$ and $\gamma_{v}$ on $R_{0}$.

\section{Numerical simulation}

This section aims to find the numerical solution of ODEs Eqs. (1)-(8) with the help of a medium order method in a finite interval of time using Matlab (Derouich and Boutayeb 2006; Khan, Ullah, and Farooq 2018; 


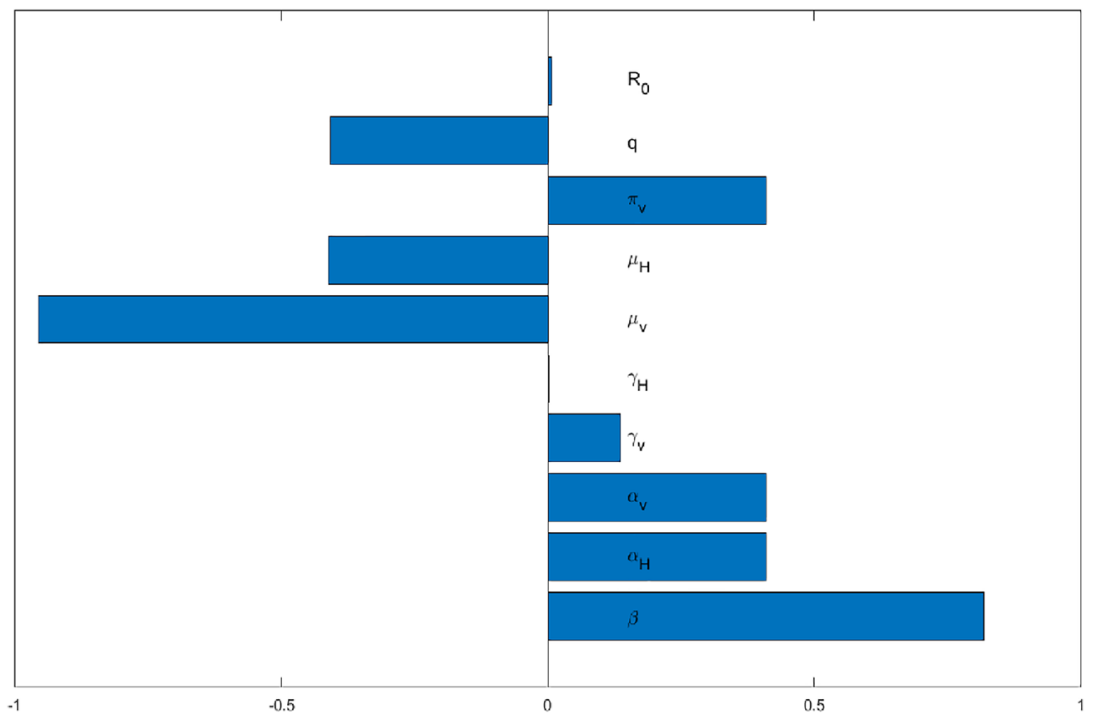

Figure 10: PRCC results for significance of parameters in $R_{0}$.

Table 4: Sensitivity indices of $R_{0}$ to the parameter values of the model.

\begin{tabular}{llr}
\hline Variable & Parameter & Sensitivity index \\
\hline$R_{0}$ & $\beta$ & 0.817806 \\
& $\alpha_{v}$ & 0.408903 \\
& $\gamma_{v}$ & 0.137216 \\
$\gamma_{H}$ & 0.189935 \\
$\mu_{v}$ & -0.955022 \\
$\mu_{H}$ & -0.412268 \\
$\pi_{v}$ & 0.408903 \\
$q$ & -0.407538 \\
\hline
\end{tabular}

Ullah et al. 2020; Ullah, Khan, and Farooq 2018b), confirming results acquired with analysis and multiple observations give a deeper understanding of the model. For this used parameter values are provided in Table 5. These parameters value satisfy the conditions for the existence of endemic equilibrium, $W_{1}$ are obtained as $S_{v}^{*}=1.932 \times 10^{7}, E_{v}^{*}=15,546.5, I_{v}^{*}=2.05214 \times 10^{6}, S_{H_{1}}^{*}=361,823.0, S_{H_{2}}^{*}=2,007,410.0, E_{H}^{*}=6228.1, I_{H}^{*}=$ $51,197.9, R_{H}^{*}=1.52836 \times 10^{7}$.

The value of basic reproduction number $\left(R_{0}\right)$ is got as 0.001835 which is less than one as our requirement. Stability conditions are also fully desired for these parameter values. The matrix's eigenvalues are obtained for $W_{01}$ as $-0.025,-0.0154$ (multiplicity 3), $-4.6130,-3.3147,-0.08589$ and -0.0140 the polynomial roots satisfied the given stability conditions. It is articulate that all the eigenvalues are negative. Similarly, for the matrix $W_{10}$, found the condition for basic reproduction number $R_{0}>3.364$ and the matrix eigenvalues are $-0.0473,-0.0154,-0.1,-0.8211,-4.6380,-3.2928,-0.0665$ and -0.0322 .

To show the nonlinear stability behaviour of disease prevalence in their respected population. For this, we have taken 110 units for susceptible vector and exposed vector, 800 units for infected vector. We plotted the solution trajectories at different initial starts using the above set of parameter values. These trajectories are shown in Figures 11, 12, and 13 respectively. In Figure 11, the variation of $S_{v}$ w.r.t the time $t$ for the different values of transmission probability $\alpha_{v}=0.55,0.65,0.75,0.85$ and 0.95 are shown properly. It suggests that the population of susceptible vectors increase with a decrease in transmission probability. Therefore, to reduce the susceptible vector population's size, we have to increase the transmission between infected mosquito to susceptible human. Also, in Figure 12, it is interesting to note that for different values of $\alpha_{v}$, the exposed vector 
Table 5: Epidemiological parameters and their values with unit.

\begin{tabular}{lll}
\hline Parameters & Values (Ghosh, Tiwari, and Chttopadhyay 2019) & Unit \\
\hline$\pi_{v}$ & 350,000 & day $^{-1}$ \\
$\beta$ & 2 & day $^{-1}$ \\
$\alpha_{H}$ & 0.75 & day $^{-1}$ \\
$\mu_{v}$ & 0.025 (Assumed) & day $^{-1}$ \\
$\gamma_{v}$ & 0.0495 (Assumed) & day $^{-1}$ \\
$\pi_{H}$ & 273,600 & day $^{-1}$ \\
$r$ & 0.25 & day $^{-1}$ \\
$\alpha_{v}$ & 0.75 & day $^{-1}$ \\
$\mu_{H}$ & 0.0154 & day $^{-1}$ \\
$\gamma_{H}$ & 3.3 & day $^{-1}$ \\
$\theta$ & 0.5 & day $^{-1}$ \\
$q$ & 4.5972 & day $^{-1}$ \\
\hline
\end{tabular}
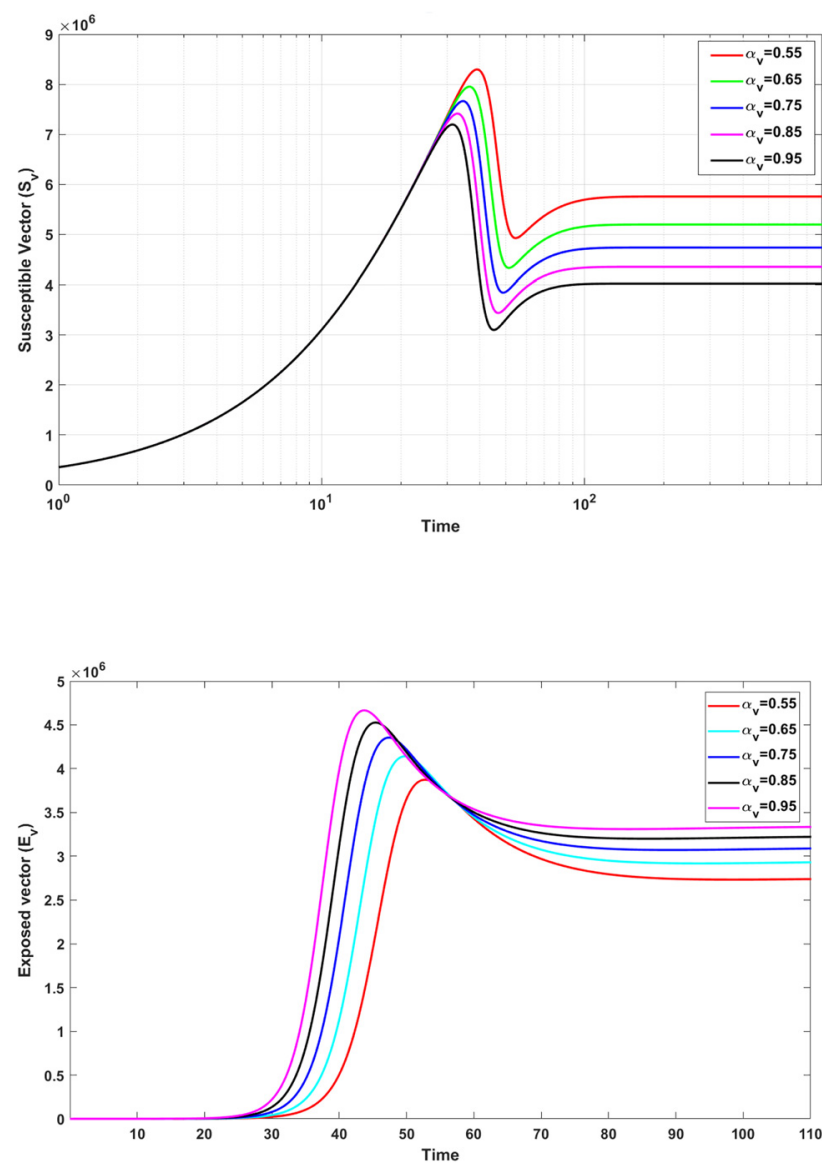

Figure 11: The plot of the susceptible vector $S_{v}$ with different value of $\alpha_{v}$.

Figure 12: The behavior of exposed vectors with $\alpha_{v}$.

population become zero in 27 unit of time whenever, if we decrease the transmission rate $\left(\alpha_{v}\right)$, it increases up to 40 unit of time. It indicates that if we decrease the rate of mosquito, the exposed vector become increases. Further, the variation of infected vector w.r.t time $t$ for different values of $\beta$ is given in Figure 13. It is evident that as the value of $\beta$ increases, the total infected population $\left(I_{v}\right)$ also increases. So, we infer that by multiple biting of mosquitoes, abundant of infected vector rapidly. 


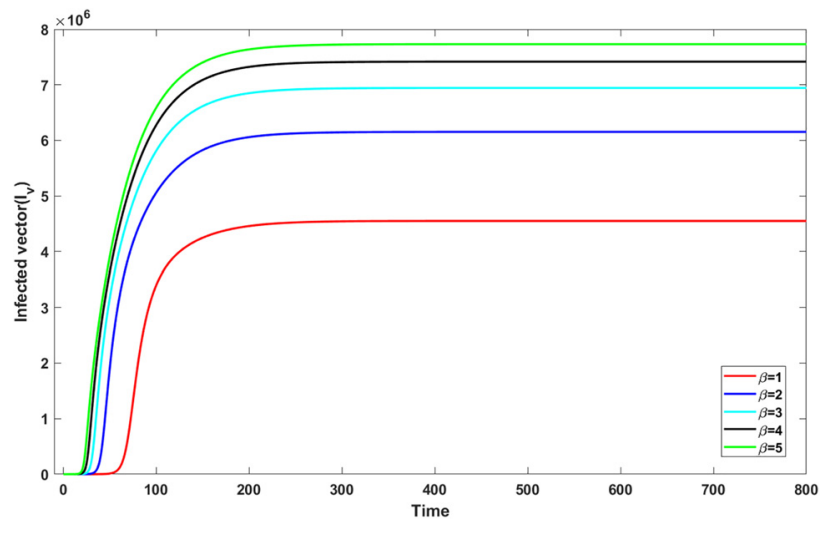

Figure 13: The total number of infected individuals for different values of $\beta$.

\section{Conclusions}

Vector-borne disease outbreaks have ruined many countries. From which, focused on assessing the dynamics of dengue fever in this article. We proposed a mathematical model, modelling them and incorporating the effect of the variable human with exponential growth. We discussed the positivity and boundedness of the system by Metzler property and found the basic reproduction number $\left(R_{0}\right)$ that control the transmission of disease and the growth rate of the infected human population. The sensitivity of $R_{0}$ obtained very sensitive for the model's parameters corresponding to transmission rate, mobilization rate of vector, biting rate, and human death rate. Numerical simulations with different parameter settings illustrate the succession of epidemics, their amplitudes and justify theoretical findings. So, in this paper, main objective is to understand the dynamics of the dengue virus with variable human and investigate the model stability criteria for the dynamics of the dengue virus. Based on stability results and the basic reproduction number, we can say that our model is very beneficial for us. Also, it discussed the sensitivity and numerical simulation of the system. Thus we can conclude that our model gives us the most reliable results on changing the nonlinear model's complexity by incorporating more epidemiological parameters, more saturating functions with respect to different virus types of the disease and mode of transmission.

According to the medical point of view, we believe that our model analysis is beneficial for humans also. It would assist in controlling the spreads of dengue disease.

Research funding: Not applicable.

Author contribution: All authors have accepted responsibility for the entire content of this manuscript and approved its submission.

Competing interest: Not applicable.

Informed consent: Not applicable.

Ethical approval: Not applicable.

\section{References}

Abate, A., A. Tiwari, and S. Sastry. 2009. “Box Invariance in Biologically-Inspired Dynamical Systems.” Automatica 45 (7): 1601-10.

Agusto, F., and M. Khan. 2018. “Optimal Control Strategies for Dengue Transmission in Pakistan.” Mathematical Biosciences 305: $102-21$.

Altaf Khana, M., S. Ullah, and M. Farooqb. 2018. "A New Fractional Model for Tuberculosis with Relapse via Atangana-Baleanu Derivative." Chaos, Solitons \& Fractals 116: 227-38.

Baniya, V., and R. Keval. 2020. "The Influence of Vaccination on the Control of JE with a Standard Incidence Rate of Mosquitoes, Pigs and Humans." Journal of Applied Mathematics and Computing 64 (1-2): 519-50. 
Bonyah, E., M. A. Khan, K. O. Okosun, and S. Islam. 2017. “A Theoretical Model for Zika Virus Transmission.” PLoS One 12 (10): e0185540.

Busenberg, S., and P. V. Driessch. 1990. "Analysis of a Disease Transmission Model in a Population with Varying Size." Journal of Mathematical Biology 28 (3): 257-70.

Derouich, M., and A. Boutayeb. 2006. “Dengue Fever: Mathematical Modelling and Computer Simulation.” Applied Mathematics and Computation 177 (2): 528-44.

Driessche, P. V. 2017. “Reproduction Number of Infection Disease Model.” Infectious Disease Modelling 2 (3): $288-303$.

Driessch, P. V., and J. Watmough. 2002. "Reproduction Number and Subthreshold Endemic Equilibria for Compartmental Models of Disease Transmission.” Mathematical Biosciences 180 (1-2): 29-48.

Esteva, L., and C. Vargas. 1998. “Analysis of a Dengue Disease Transnmission Model.” Mathematical Biosciences 150 (2): 131-51.

Esteva, L., and C. Vargas. 1999. “A Model for Dengue Disease with Variable Human Population.” Journal of Mathematical Biology 38 (3): $220-40$.

Esteva, L., and C. Vargas. 2009. "Influence of Vertical and Mechanical Transmission on the Dynamics of Dengue Disease." Mathematical Biosciences 167 (1): 51-64.

Ghosh, I., P. K. Tiwari, and J. Chttopadhyay. 2019. "Effect of Active Case Finding on Dengue Control: Implication From a Mathematical Model." Journal of Theoretical Biology 464: 50-62.

Kar, T. K., and S. Jana. 2009. "Application of Three Controls Optimally in a Vector-Borne Disease- A Mathematical Study." Communications in Nonlinear Science and Numerical Simulation 18 (10): 2868-84.

Khan, M. A., and A. Atangana. 2020. "Modeling the Dynamics of Novel Corona-Virus (2019-nCov) with Fractional Derivative." Alexandria Engineering Journal 59 (4): 2379-89.

Khan, M. A., Z. Hammouch, and D. Baleanu. 2019. "Modeling the Dynamics of Hepatitis E via the Caputo-Fabrizio Derivative." Mathematical Modelling of Natural Phenomena 14 (3): 311.

Khan, M. K., S. Ullah, and M. Farooq. 2018. "A New Factional Model for Tuberculosis with Relapse via Atangana-Baleanu Derivative." Chaos, Solitons \& Fractals 116: 227-38.

Lakshmikantham, V., S. Leela, and A. A. Martynyuk. 1989. Stability analysis of Nonlinear Systems. New York: Marcel Dekker.

Linda, J. A., and M. B. Amy. 2000. "Comparison of Deterministic and Stochastic SIS and SIR Models Discrete-Time." Mathematical Biosciences 163 (1): 1-33.

Misra, A., and A. Sharma. 2013. "A Mathematical Model For Control of Vector-Borne Disease through Media Campaigns." Discrete \& Continuous Dynamical Systems - Series B 18 (7): 1909-27.

Nur, W., H. Rachman, and M. Abdal. 2018. "SIR Model Analysis For Transmission of Dengue Fever Disease With Climate Lyapunov Function.” Journal of Physics: Conference Series 1028: 012117.

Panja, P., K. Mondal, and J. Chattopadhyay. 2018. "Stability, Bifurcation and Optimal Control Analysis of a Malaria Model in a Periodic Environment.” International Journal of Nonlinear Sciences and Numerical Simulation 19 (6): 627-42.

Syafruddin, S., and M. Noorani. 2013. "Lyapunov Function of SIR and SEIR Model for Transmission of Dengue Fever Disease." International Journal of Simulation and Process Modelling 8 (2/3): 177.

Tewa, J., J. L. Dimi, and S. Bowong. 2009. “Lyapunov Function for a Dengue Disease Transmission Model.” Chaos, Solitons \& Fractals 39: 936-41.

Ullah, S., M. A. Khan, M. Farooq, and E. O. Alzahrani. 2020. “A Fractional Model for the Dynamics of Tuberculosis (TB) using Atangana-Baleanu derivative." Discrete \& Continuous Dynamical Systems - Series B 13 (3): 937-56.

Ullah S., M. A. Khan, and M. Farooq. 2018a. "Modelling and Analysis the Fractional HBV Model with Atangana- Baleanu Derivative.” The European Physical Journal Plus 133 (8), https://doi.org/10.1140/epjp/i2018-12120-1.

Ullah S., M. A. Khan, and M. Farooq. 2018b. "A New Fractional Model for the Dynamics of Hepatitis B Virus Using Caputo-Fabrizio Derivative.” The European Physical Journal Plus 133 (6), https://doi.org/10.1140/epjp/i2018-12072-4.

WHO. 2020. Dengue Control. World Health Organization. https://www.who.int/denguecontrol/human/en.

Zheng, T., and L. Nie. 2018. "Modelling the Transmission Dynamics of Two-Strain Dengue In the Presence Awareness and Vector Control." Journal of Theoretical Biology 443: 82-91.

Zhu, M., and Y. Xu. 2019. “A Time-Periodic Dengue Fever Model in a Heterogeneous Enviroment.” Mathematics and Computers in Simulation 155: 115-29. 